\title{
Ações de educação em higienização das mãos como estratégia à segurança do paciente: Relato de experiência
}

\section{Education actions on hand hygiene as a strategy for patient safety: Experience report}

\author{
Elaine Cristina Bezerra Almeida \\ Enfermeira especialista em Qualidade e Segurança do Paciente, Santa Casa de Misericórdia de Sobral, elainecrisbezerra@ hotmail.com \\ Antonio Neudimar Bastos Costa \\ Farmacêutico especialista em Farmácia Clínica e Cuidados Farmacêuticos, Santa Casa de Misericórdia de Sobral, neudimar.bastos@gmail.com \\ Patricia Batista Rosa \\ Médica Infectologista, Santa Casa de Misericórdia de Sobral, rosapb@uol.com.br \\ Cristiano Araújo Costa \\ Médico Diretor Técnico, Santa Casa de Misericórdia de Sobral, cristiano@stacasa.com.br \\ Tiago Sousa Melo \\ Farmacêutico Doutor em Biotecnologia, Instituto Nacional de Teologia Aplicada, tiagosousam@gmail.com
}

\begin{abstract}
Resumo: Objetivou-se relatar a experiência de ações de educação em serviço da Comissão de Controle de Infecção com o intuito de sensibilizar a equipe multiprofissional da assistência em saúde com relação à adoção de práticas seguras de higienização das mãos de acordo com a Agência Nacional de Vigilância Sanitária. Assim, foi desenvolvida intervenção de higienização das mãos na Unidade de Terapia Intensiva Adulta. Foram discutidos a importância da prática de higienização das mãos para a prevenção e o controle de infecções relacionadas à assistência à saúde e a capacitação de profissionais. Nesse contexto, visando a educação em saúde foram utilizados cartazes e panfletos da Agência Nacional de Vigilância Sanitária, almejando as melhores práticas voltadas à segurança do paciente na busca de despertar nesse público, o interesse de desenvolver e/ou permanecer com a prática de segurança do paciente. Considera-se haver discussão sobre o tema por parte dos profissionais para superação de desafios. As ações educativas constituem importante ferramenta para o direcionamento de ações que visem o desenvolvimento de melhorias no cuidado em saúde.
\end{abstract}

Palavras-chave: Infecção hospitalar; Controle de infecções; Qualidade da assistência à saúde.

\begin{abstract}
It was aimed to report the experience of actions of education in service of Infection Control Committee in intention to sensitize the multiprofissional healthcare team about the adoption of safe hand hygiene practices according to the Brazilian Health Surveillance Agency. Thus, was developed a hand hygiene intervention in adult Intensive Care Unit. The importance of hand hygiene practice for prevention and control health care-associated infections and professional training are discussed. In this context, posters and pamphlets of Brazilian Health Surveillance Agency aiming better practices designed to patient safety were used with the purpose of fostering to health education and in order to awaken interest in the public to develop and/or to remain with the safety patient practice. It was considered there must be discussion about the subject by professionals to overcome challenges. Educational actions are an important tool for directing actions aimed at developing improvements in health care.
\end{abstract}

Keywords: Cross infection; Infection control; Quality of health care. 


\section{INTRODUÇÃO}

Desde 1846 a higienização das mãos é reconhecida como a medida mais eficaz na prevenção das infecções relacionadas à assistência à saúde (IRAS) e considerada a mais importante ação para reduzir a transmissão de infecções nos serviços de saúde (WHO, 2009). Ressalta-se que esta simples medida visa à segurança do paciente, dos profissionais de saúde e de todos aqueles envolvidos nos cuidados aos pacientes (CDC, 2002).

A Agência Nacional de Vigilância Sanitária (ANVISA) afirma que as infecções estão associadas a um aumento da morbidade, mortalidade, tempo de internação e, consequentemente, custos com o tratamento (ANVISA, 2009). Segundo a Organização Mundial da Saúde (OMS), mais de 1,4 milhões de pessoas desenvolvem algum tipo de IRAS em Unidades de Terapia Intensiva. De cada 100 pacientes hospitalizados em qualquer momento, $7 \mathrm{em}$ países desenvolvidos e 10 em países em desenvolvimento adquiriram IRAS. Milhões de pacientes são afetados em todo o mundo a cada ano. Medidas simples e de baixo custo de prevenção e controle de infecção, como a higiene das mãos, podem reduzir a frequência de infecções em mais de 50\% (WHO, 2014).

Ainda, as IRAS afetam cerca de $30 \%$ dos pacientes e a mortalidade atribuível a elas pode chegar a $44 \%$ nos serviços de saúde, sendo as mais frequentes a infecção do trato urinário, a infecção do sítio cirúrgico, a infecção da corrente sanguínea e a infecção do trato respiratório (WHO, 2009b).

Esse problema agrava-se em Unidade de Terapia Intensiva (UTI), nas quais as taxas de infecções hospitalares, em relação às demais unidades de um hospital, é geralmente causado por pacientes com suscetibilidade aumentada a infecções, tanto pelo estado clínico, como pelos procedimentos invasivos e risco aumentados de transmissão de microorganismos entre pacientes (BRASIL, 2004)

Nesse contexto, as iniciativas com o objetivo de mitigar as IRAS pelo Ministério da Saúde, como um manual com informações para os profissionais de saúde, a fim de orientá-los quanto às normas e aos procedimentos para lavar as mãos, visando à prevenção e o controle das infecções, e a divulgação, pelo Centro de Controle de Doença e Prevenção (CDC) do guia para higiene de mãos em serviços de assistência à saúde (BRASIL, 2009). Nesta última publicação, o termo lavagem das mãos foi substituído por higienização das mãos devido à maior abrangência desse procedimento. De acordo com esse documento, a higienização das mãos é um termo geral que se refere a qualquer ação de higienizar as mãos para prevenir a transmissão e consequentemente evitar e controlar as IRAS.

Além dessas, a OMS por meio da Aliança Mundial para a Segurança do Paciente, tem dedicado esforços no sentido de elaborar diretrizes e estratégias para a implantação de medidas visando à adesão dos profissionais de saúde às práticas de higienização das mãos (ANVISA, 2009). Essa iniciativa está direcionada para os serviços de saúde, envolvendo os profissionais, os pacientes e a comunidade, com o objetivo de reduzir os riscos inerentes às IRAS (WHO, 2006a; WHO, 2006b).
Segundo Pedreira (2011) e WHO (2014), a segurança do paciente é determinada como à redução dos riscos de danos desnecessários ao paciente durante todos os procedimentos de cuidado, e abrange em seu contexto a promoção de uma assistência em saúde até um mínimo aceitável, no momento certo, que seja equânime e fundamentada na melhor evidência científica e nas necessidades integrais e individuais do paciente e de sua família. Nessa conjuntura, a importância da higienização das mãos tem se tornado uma prioridade para os gestores de serviços de saúde do século XXI, especialmente devido às IRAS causadas por microorganismos multirresistentes de relevância epidemiológica (CDC, 2002).

Nesse sentido, considerando que as infecções hospitalares constituem um grave problema de saúde pública aos usuários dos hospitais, e sua prevenção e controle envolvem critérios de adequação da assistência hospitalar, da vigilância sanitária e outras, nas esferas do Estado, do Município e de cada hospital, atinentes a seu funcionamento. Em 1998 foi instituída a portaria 2.616 que dispõe sobre a obrigatoriedade da manutenção pelos hospitais do país, de Comissões de Controle de Infecções Hospitalares (CCIH's), com vistas à redução máxima possível da incidência e da gravidade das infecções hospitalares (BRASIL, 1998).

Recentemente, com a publicação do programa nacional de segurança do paciente, a higienização das mãos tem se destacado ainda mais como medida primária e eficaz de prevenção das IRAS (BRASIL, 2013). Dessa forma, é imprescindível que todos os serviços de saúde cumpram o seu papel, priorizando as práticas de higienização das mãos, oferecendo assistência segura, de acordo com as necessidades de saúde do paciente, e se preocupando também com a minimização de riscos.

Assim, objetivou-se com presente trabalho relatar a experiência de ação de educação em serviço, com o intuito de sensibilizar a equipe multiprofissional da assistência em saúde, com relação à adoção de práticas seguras de higienização das mãos de acordo com a ANVISA.

\section{MATERIAL E MÉTODOS}

Este trabalho caracteriza-se como um estudo descritivo, do tipo relato de experiência desenvolvido na UTI Adulto da Santa Casa de Misericórdia de Sobral (SCMS) no Ceará e realizado no mês de outubro de 2016.

A SCMS é um hospital filantrópico sem fins lucrativos que presta serviços de alta, média e baixa complexidade, como, neurocirurgia, urologia, hemodiálise, traumato-ortopedia, pediatria, quimioterapia, oncologia, esterilização.

A Instituição é um hospital de ensino e de referência da região noroeste do Estado do Ceará. Atualmente, possui 92 anos e atende a cinquenta e sete municípios da macrorregião.

O treinamento foi realizado pela Comissão de Controle de Infecção Hospitalar (CCIH) e teve caráter educativo e de conscientização, não foram adotadas medidas punitivas para os profissionais como advertências, suspensões ou denúncia de infração ética.

Inicialmente, a $\mathrm{CCIH}$ realizou uma reunião com os coordenadores, médico e equipe de enfermagem da UTI para combinar o dia e o horário mais apropriados para o cumprimento da ação. Em seguida, foram entregues 
convites aos profissionais de saúde com a data e horário acertados.

Após reunião da Comissão definiu-se que a capacitação seria realizada em um espaço dentro da UTI para que todos os profissionais diretamente envolvidos na assistência pudessem participar do momento. A qualificação ocorreu nos três turnos de trabalho e em três dias, dois dias da semana e um dia no fim de semana.

Optou-se como estratégia a exposição de cartazes e panfletos padronizados pela ANVISA, além de treinamento prático de higienização das mãos. Alguns resultados de artigos, revisões de literatura que foram repassados para ratificar a importância dessa medida para a prevenção e o controle de IRAS.

Caso alguma irregularidade fosse encontrada, o profissional que contraveio às normas de precaução e o coordenador responsável pela unidade eram orientados quanto às práticas adequadas (FIGUEIREDO, 2010).

No contexto, salienta-se que a medida primordial para a segurança do paciente é enfatizar a importância da higienização das mãos por todos os profissionais de saúde, visitantes e acompanhantes para evitar a transmissão cruzada de IRAS nos serviços de saúde.

\section{RESULTADOS E DISCUSSÃO}

Apesar da relevância da higienização das mãos para a prevenção das infecções hospitalares, a adesão a esse procedimento tem se constituído em um enorme desafio para as CCIH's das instituições de saúde. Dentre vários aspectos a serem trabalhados, inclui-se prioritariamente os profissionais, necessitando de capacitação, de conscientização, de estratégias de uso e racionalização dos insumos (NEVES et al., 2006).

Nesse sentido, tentando aumentar a adesão à prática de higienização das mãos buscou-se envolver toda a equipe multiprofissional e verificou-se uma significativa colaboração dos profissionais de saúde do setor. Isso tornou o momento de educação mais dinâmico e participativo, potencializando a disseminação do conhecimento, tornando-o igualitário e efetivo. Nos três turnos, todos os profissionais que estavam de plantão participaram do momento, desde a chegada da CCIH ao setor, todos demonstraram interesse em participar. Houve, em alguns instantes, interrupções quando necessário para a realização de procedimento nos pacientes, no entanto não ocorreu prejuízo ao profissional.

Diversos motivos são apontados para a baixa participação dos profissionais para à higienização das mãos, destacando-se a falta de motivação, ausência de pias próximas ao paciente e de recursos materiais adequados, reações cutâneas nas mãos, falta de tempo e até mesmo a falta de informação sobre a importância das mãos na contaminação cruzada (OLIVEIRA et al., 2010).

Neste estudo, o principal motivo para o descumprimento da higienização das mãos foi o desconhecimento de alguns profissionais de saúde quanto a importância do cumprimento dessa medida para a prevenção e o controle de IRAS. A maioria dos profissionais afirmou que não acreditava que só a higienização das mãos fosse capaz de prevenir as infecções. Constatou-se, nesse momento que esses profissionais não sabiam que o modo de transmissão das bactérias é através das mãos. Muitos desses acreditavam que a utilização de máscaras seria o suficiente. Nesse momento, houve a orientação e desmistificação de que as bactérias poderiam ser transmitidas pelo ar. Dessa forma, a experiência sinalizou a relevância da educação em serviço para a adesão a novas e adequadas posturas.

Outro ponto importante que pode ser esclarecido aos profissionais do setor foi quanto a necessidade de ter cartazes informativos próximo as pias e aos dispensadores de álcool em gel a fim de facilitar o cumprimento correto da higienização. Até o momento a CCIH notava uma resistência quanto a permanência dos cartazes de higienização das mãos no setor. Após os esclarecimentos, no entanto, a equipe entendeu que a distribuição de materiais educativos na unidade, também poderia ser um fator positivo para a redução do risco de infecção.

Verifica-se que, para que exista o cumprimento das normas, é preciso haver conhecimento técnico sobre a maneira adequada de higienizar as mãos (FIGUEIREDO, 2010). Dessa maneira, pode-se perceber a importância desse momento para além de uma atividade de educação em saúde, mas como uma ferramenta de otimização de novas práticas pelos trabalhadores do serviço.

Foi evidenciada a importância dessas atividades com maior frequência de modo a contemplar um maior número de profissionais devido à rotatividade dos mesmos. Destacou-se também, a necessidade de ampliar as ações pelas demais unidades do hospital.

Essas discussões acerca de medidas essenciais à segurança do paciente merecem ser cada vez mais impulsionadas nas formações acadêmicas (SOUSA et al., 2012).

Dados da literatura mostram que a abordagem da temática segurança do paciente no currículo dos cursos de graduação é mais frequente em países desenvolvidos, como nos Estados Unidos da América (EUA) e no Reino Unido, e em geral ocorre como disciplina optativa, não havendo inclusão formal na estrutura curricular educacional em saúde (SOUSA, 2014). Nesse sentido, educação em saúde traz desafios à formação de alunos sensibilizados, às potencialidades de uma prática em que o cuidar e o educar andam juntos (SOUSA et al., 2012). No entanto, a relação existente entre teoria e a prática não significa, maior seguimento das normas estabelecidas, pois há vários motivos que podem dificultar a adesão como: a ausência de motivação, o despreparo dos profissionais, sobrecarga de trabalho e o comportamento inadequado de membros mais experientes influenciando negativamente os demais profissionais da equipe, dentre outros (LOPES et al., 2008).

Assim, percebe-se que as atividades de educação permanente e continuada devem motivar a equipe que trabalha em serviços de saúde desenvolvendo ações locais que reforcem a higienização das mãos, como medida primordial para a prevenção e controle de IRAS. Desse modo, a construção do conhecimento deve ser pautada na vivência de experiências significativas, numa abordagem dialógica (FIGUEIREDO, 2010). Nesse sentido, o desenvolvimento de programas educativos com o objetivo de treinar ou reciclar deve ser rotina da unidade, avaliando criteriosamente os resultados sobre a adesão dos profissionais de saúde às medidas de precaução instruídas, aprimorando as estratégias quando necessário (LOPES et al., 1999). 


\section{CONCLUSÕES}

Há necessidade de ter uma equipe consciente das medidas de prevenção de infecção, como forma de tornar a assistência mais segura.

A construção do conhecimento deve ser pautada na vivência dos profissionais, permitindo a aproximação entre educador e o educando por meio do diálogo. Assim, surge estratégias educativas implementadas que sejam avaliadas periodicamente, buscando sua melhoria e maior eficácia. Com isso, a educação permanente em saúde é uma importante estratégia pedagógica e constitui-se como uma ferramenta fundamental na atualização das práticas dos profissionais de saúde. Dessa forma, a educação permanente é uma medida essencial para o desenvolvimento de melhorias no cuidado em saúde capaz de proporcionar ao trabalhador o pensar sobre o seu trabalho e adequar-se a rotinas corretas.

Para enfrentar o problema das infecções relacionadas à assistência à saúde, garantindo a segurança do paciente é imprescindível a contribuição de todos, desde os pacientes, os profissionais e até os tomadores de decisões políticas, de modo que sejam parte da solução do problema.

\section{REFERÊNCIAS}

BRASIL. Ministério da Saúde. Portaria no . 2.616, de 12 de Maio de 1998. Regulamenta as ações de controle de infecção hospitalar no país. Diário Oficial da República Federativa do Brasil, 15 maio 1998. Seção I.

BRASIL. Agência Nacional de Vigilância Sanitária. Módulo 4: PREVENÇÃO DE INFECÇÕES EM UNIDADE DE TERAPIA INTENSIVA. Versão 1.0; São Paulo. 2004.

BRASIL. Agência Nacional de Vigilância Sanitária. Segurança do Paciente em Serviços de Saúde: Higienização das Mãos / Agência Nacional de Vigilância Sanitária. Brasília: Anvisa, 2009. 105p.

BRASIL. Ministério da Saúde. Portaria $n^{\circ}$. 529, de 1 de abril de 2013. Institui o Programa Nacional de Segurança do Paciente (PNSP). Diário Oficial da União, Brasília, 1 de abril de 2013. Seção I.

CDC, CENTERS FOR DISEASE CONTROL AND PREVENTION. Guideline for hand hygiene in health-care settings: recommendations of the Healthcare Infection Control Practices Advisory Committee and the HICPAC/SHEA/APIC/IDSA Hand Hygiene Task Force. MMWR Recomm Rep, Atlanta, v. 51, n. RR-16, p. 1-45, Oct. 2002.

WHO, WORLD HEALTH ORGANIZATION. The WHO Guidelines on Hand Hygiene in Health Care (Advanced Draft). Global Patient Safety Challenge 2005-2006: Clean care is safer care. Geneva: WHO Press, 2006a. 205 p.
WHO, WORLD HEALTH ORGANIZATION. World Alliance for Patient Safety. Forward Programme 20062007. Genebra: WHO Press, 2006b. 56 p.

WHO, WORLD HEALTH ORGANIZATION. The WHO Guidelines on Hand Hygiene in Health Care. Genebra: WHO, 2009a.

WHO, WORLD HEALTH ORGANIZATION. The WHO Guidelines on Hand Hygiene in Health Care. First global patient safety challenge. Clean care is safer care. Genebra: WHO, 2009b.

WHO, WORLD HEALTH ORGANIZATION. 10 facts on patient safety. Genebra: WHO, 2014. Acesso em: 07 de abril de 2017.

FIGUEIREDO, M. F. Inspeção de Precaução de Contato: Relato de Experiência de uma Graduanda de Enfermagem. Universidade Estadual Paulista. São Paulo, 2010.

SOUSA, E. F. R.; COSTA, E. A. O.; SILVEIRA, M. A.; WERNET, M.; CAGNIN, E. R. G.; DUPAS, G. Relato de experiência do trabalho com educadores como estratégia de cuidado à saúde da criança. Revista da Sociedade Brasileira de Enfermagem Pediátrica. São Paulo,v.12, n.1, p. $49-53,2012$

SOUSA, P. Segurança do Paciente: Conhecendo os riscos nas organizações de saúde./ Organizado por Paulo Sousa e Walter Mendes. - Rio de Janeiro, EAD/ENSP, 2014. 452 p.

LOPES, A. C. S.; OLIVEIRA, A. C.; SILVA, J. T.; PAIVA, M. H. R. S. Adesão às precauções padrão pela equipe do atendimento pré-hospitalar móvel de Belo Horizonte. Caderno de Saúde Pública. Rio de Janeiro,v.4. n.6. p. 1387-96., 2008.

LOPES, M. H. B. M.; MOROMIZATO, S. S.; VEIGA, J. F. F. S. Adesão às medidas de precaução-padrão: relato de experiência. Revista Latino Americana de Enfermagem. v.7. n.4, p.83-88. Ribeirão Preto, 1999.

NEVES, Z. C. P.; TIPPLE, A. F. V.; SOUZA, A. C. S.; PEREIRA, M. S.; MELO, D. S.; FERREIRA, L. R. Higienização das mãos: o impacto de estratégias de incentivo a adesão entre profissionais de saúde de uma unidade de terapia intensiva neonatal.Rev Latino-am Enferm,2006.

OLIVEIRA, A. C.; CARDOSO, A. S. MASCARENHAS, D. Precauções de contato em unidades de terapia intensiva: agentes facilitadores e dificultadores para adesão de profissionais. Revista Esc. Enf USP, 2010;44(1):161-5.

PEDREIRA, M. L. G. Segurança do paciente na terapia intravenosa. In: Harada M. J. C. S.; Pedreira M. L. G.; Terapia intravenosa e infusões. São Caetano do Sul, (SP): Yendis; 2011 p.15-29. 